\title{
Global optimality results for multivalued non-self mappings in b-metric spaces
}

\author{
Moosa Gabeleh $^{1} \cdot$ Robert Plebaniak $^{2}$
}

Received: 24 February 2016 / Accepted: 15 February 2017 / Published online: 27 February 2017

(C) The Author(s) 2017. This article is published with open access at Springerlink.com

\begin{abstract}
In this paper, we introduce a new class of multivalued contractions with respect to b-generalized pseudodistances and prove a best proximity point theorem for this class of non-self mappings. In this way, we improve and extend several existing results in the literature. Examples are given to support our main results. This work is a continuation of studies on the use of a new type of distances in the fixed point theory. The pioneering effort in direction of defining distance is inter alia paper of O. Kada, T. Suzuki and W. Takahashi.
\end{abstract}

Keywords Best proximity point $\cdot$ Multivalued contraction of Suzuki type $\cdot$ b-Generalized pseudodistances

Mathematics Subject Classification $47 \mathrm{H} 10 \cdot 47 \mathrm{H} 09 \cdot 46 \mathrm{~B} 20$

\section{Introduction}

In 2008, Suzuki [1] presented a weaker notion of contractions in order to characterize the completeness of metric spaces and established the following interesting theorem.

Theorem 1.1 (Suzuki [1]) Let $(X, d)$ be a complete metric space and let $T$ be a self-mapping on $X$. Define a nondecreasing function $\theta:[0,1) \rightarrow\left(\frac{1}{2}, 1\right]$ by

Robert Plebaniak

robpleb@math.uni.lodz.pl

Moosa Gabeleh

gab.moo@gmail.com

1 Department of Mathematics, Ayatollah Boroujerdi University, Boroujerd, Iran

2 Department of Nonlinear Analysis, Faculty of Mathematics and Computer Science, University of Łódź, Banacha 22, 90-238 Lodz, Poland 


$$
\theta(r)= \begin{cases}1 & \text { if } 0 \leq r \leq \frac{1}{2}(\sqrt{5}-1) \\ \frac{1-r}{r^{2}} & \text { if } \frac{1}{2}(\sqrt{5}-1) \leq r \leq \frac{1}{\sqrt{2}} \\ \frac{1}{1+r} & \text { if } \frac{1}{\sqrt{2}} \leq r<1\end{cases}
$$

Then for a metric space $(X, d)$, the following are equivalent:

(i) $X$ is complete.

(ii) There exists $r \in[0,1)$ such that every mapping $T$ on $X$ satisfying the following:

$$
\theta(r) d(x, T x) \leq d(x, y) \text { implies } d(T x, T y) \leq r d(x, y), \forall x, y \in X,
$$

has a fixed point.

After that the multivalued version of Theorem 1.1, which is an extension of Nadler's fixed point theorem, was presented as below.

Theorem 1.2 [2] Define a strictly decreasing function $\eta$ from $[0,1)$ onto $\left(\frac{1}{2}, 1\right]$ by

$$
\eta(r)=\frac{1}{1+r} .
$$

Let $(X, d)$ be a complete metric space and let $T: X \rightarrow 2^{X}$ be a multivalued mapping such that $T(x)$ is a nonempty, bounded and closed subset of $X$ for each $x \in X$. Assume that there exists $r \in[0,1)$ such that

$$
\eta(r) \mathcal{D}(x, T x) \leq d(x, y) \text { implies } \mathcal{H}(T x, T y) \leq r d(x, y),
$$

for all $x, y \in X$, where $\mathcal{H}$ denotes the Hausdorff metric. Then there exists $z \in X$ such that $z \in T z$.

Now, let $(A, B)$ be a nonempty pair of subsets of a metric space $(X, d)$ and let $T: A \rightarrow 2^{B}$ be a multivalued non-self mapping. Then for each $x \in A$ we have $\mathcal{D}(x, T x) \geq \operatorname{dist}(A, B)$, where $\operatorname{dist}(A, B):=\inf \{d(x, y):(x, y) \in A \times B\}$ and $\mathcal{D}(x, T x):=\operatorname{dist}(\{x\}, T x)$. So, it is quite natural to seek an approximate solution $x \in A$ that is optimal in the sense that the distance $\mathcal{D}(x, T x)$ with respect to $\mathcal{D}$ is minimum. As the minimality of the value $\mathcal{D}(x, T x)$ connotes the highest closeness between the elements $x$ and $T x$, one attempts to determine an element $x$ for which $\mathcal{D}(x, T x)$ assumes the least possible value $\operatorname{dist}(A, B)$. Such an optimal solution $x$ for which $\mathcal{D}(x, T x)=\operatorname{dist}(A, B)$, is called a best proximity point of the multivalued non-self mapping $T$. Existence of best proximity points for multivalued nonself mappings was first studied in [3] for multivalued nonexpansive non-self mappings in hyperconvex metric spaces and in Hilbert spaces (see also [4-10] for different approaches to the same problem).

The aim of this article is to elicit a best proximity point theorem for a new class of multivalued non-self mappings with respect to $b$-generalized pseudodistances. Our results improve and extend some recent results in the previous works.

\section{Preliminaries}

Let $A$ and $B$ be two nonempty subsets of a metric space $(X, d)$. When we say that a pair $(A, B)$ satisfies a special property, we mean that both $A$ and $B$ satisfy the mentioned property. 
We denote by $\mathcal{C B}(X)$ the family of all nonempty closed bounded subsets of $X$. We will use the following notations:

$$
\begin{aligned}
& \mathcal{D}^{*}(a, B)=\mathcal{D}(a, B)-\operatorname{dist}(A, B), \quad \forall a \in A, \\
& \mathcal{H}(A, B)=\max \left\{\sup _{x \in A} \mathcal{D}(x, B), \sup _{y \in B} \mathcal{D}(y, A)\right\} \quad \forall A, B \in \mathcal{C B}(X), \\
& A_{0}=\{x \in A: d(x, y)=\operatorname{dist}(A, B) \text { for some } y \in B\}, \\
& B_{0}=\{y \in B: d(x, y)=\operatorname{dist}(A, B) \text { for some } x \in A\} .
\end{aligned}
$$

It is easy to see that if $(A, B)$ is a nonempty weakly compact pair in a Banach space $X$ then $\left(A_{0}, B_{0}\right)$ is a nonempty pair.

Definition 2.1 [11] Let $(A, B)$ be a pair of nonempty subsets of a metric space $(X, d)$ with $A_{0} \neq \emptyset$. The pair $(A, B)$ is said to have P-property if and only if

$$
\left\{\begin{array}{l}
d\left(x_{1}, y_{1}\right)=\operatorname{dist}(A, B) \\
d\left(x_{2}, y_{2}\right)=\operatorname{dist}(A, B)
\end{array} \Rightarrow d\left(x_{1}, x_{2}\right)=d\left(y_{1}, y_{2}\right),\right.
$$

where $x_{1}, x_{2} \in A_{0}$ and $y_{1}, y_{2} \in B_{0}$.

The following notion is weaker than the notion of P-property which was first introduced in [12].

Definition 2.2 [12] Let $(A, B)$ be a pair of nonempty subsets of a metric space $(X, d)$ with $A_{0} \neq \emptyset$. The pair $(A, B)$ is said to have WP-property if and only if

$$
\left\{\begin{array}{l}
d\left(x_{1}, y_{1}\right)=\operatorname{dist}(A, B) \\
d\left(x_{2}, y_{2}\right)=\operatorname{dist}(A, B)
\end{array} \quad \Rightarrow d\left(x_{1}, x_{2}\right) \leq d\left(y_{1}, y_{2}\right),\right.
$$

where $x_{1}, x_{2} \in A_{0}$ and $y_{1}, y_{2} \in B_{0}$.

Example 2.1 [11] Let $(A, B)$ be a nonempty, closed and convex pair of subsets of a Hilbert space $\mathbb{H}$. Then $(A, B)$ satisfies the P-property.

Example 2.2 Let $(A, B)$ be a nonempty pair of subsets of a metric space $(X, d)$ such that $A_{0} \neq \varnothing$ and $\operatorname{dist}(A, B)=0$. Then $(A, B)$ has the P-property.

Example 2.3 [13] Let $(A, B)$ be a nonempty bounded, closed and convex pair of subsets of a uniformly convex Banach space $X$. Then $(A, B)$ has the P-property.

Example 2.4 Consider $X:=\mathbb{R}$ with the usual metric. Suppose that

$$
A:=[1,2] \text { and } B:=\{-1,0,3\} \text {. }
$$

Then we have $\operatorname{dist}(A, B)=1$ and $A_{0}=\{1,2\}, B_{0}:=\{0,3\}$. If $\left(x_{1}, x_{2}\right)=(1,2)$ and $\left(y_{1}, y_{2}\right)=(0,3)$, then

$$
d\left(x_{1}, y_{1}\right)=d\left(x_{2}, y_{2}\right)=\operatorname{dist}(A, B) \text { and } d\left(x_{1}, x_{2}\right)<d\left(y_{1}, y_{2}\right),
$$

which deduces that $(A, B)$ has the WP-property. Note that $(B, A)$ does not have the WPproperty and so, $(A, B)$ does not have the P-property.

Here, we state the next best proximity point theorem which is a main result of [14]. 
Theorem 2.3 [14] Define a strictly decreasing function $\eta$ from $[0,1)$ onto $\left(\frac{1}{2}, 1\right]$ by

$$
\eta(r)=\frac{1}{1+r} .
$$

Let $(A, B)$ be a pair of nonempty closed subsets of a complete metric space $(X, d)$ such that $A_{0} \neq \emptyset$ and $(A, B)$ satisfies the P-property. Let $T: A \rightarrow 2^{B}$ be a multivalued non-self mapping. Assume that there exists $r \in[0,1)$ such that

$$
\eta(r) \mathcal{D}^{*}(x, T x) \leq d(x, y) \text { implies } \mathcal{H}(T x, T y) \leq r d(x, y),
$$

for all $x, y \in A$. If $T(x) \in \mathcal{C B}(X)$ for all $x \in A$, and $T\left(x_{0}\right) \subset B_{0}$ for each $x_{0} \in A_{0}$, then $T$ has a best proximity point in $A$.

The notion of b-metric space was introduced by Czerwik [15] as below.

Definition 2.4 [15] Let $X$ be a nonempty set and $s \geq 1$ be a given real number. A function $d: X \times X \rightarrow[0, \infty)$ is $b$-metric if for any $x, y, z \in X$ the following three conditions are satisfied:

$\left(d_{1}\right) d(x, y)=0 \Leftrightarrow x=y$;

$\left(d_{2}\right) d(x, y)=d(y, x)$;

$\left(d_{3}\right) d(x, z) \leq s[d(x, y)+d(y, z)]$.

If $d$ is a b-metric on $X$ (with constant $s \geq 1$ ), then the pair $(X, d)$ is called a b-metric space. Note that every metric space is a b-metric space. Throughout this paper, we assume that the $b$-metric $d: X \times X \rightarrow[0, \infty)$ is continuous on $X^{2}$.

Here, we mention the following fixed point theorem which is the main result of [15].

Theorem 2.5 [15] Let $(X, d)$ be a complete b-metric space and $T: X \rightarrow \mathcal{C B}(X)$ be a multivalued mapping. Suppose there exists $r \in\left(0, \frac{1}{s}\right)$ so that

$$
\mathcal{H}(T x, T y) \leq \operatorname{rd}(x, y)
$$

for all $x, y \in X$. Then $T$ has a fixed point.

Definition 2.6 [16] Let $X$ be a $b$-metric space (with constant $s \geq 1$ ). The map $J: X \times X \rightarrow$ $[0, \infty)$, is said to be a $b$-generalized pseudodistance on $X$ if the following two conditions hold:

$(J 1) J(x, z) \leq s[J(x, y)+J(y, z)]$ for any $x, y, z \in X$; and

$(J 2)$ For any sequences $\left(x_{m}: m \in \mathbb{N}\right)$ and $\left(y_{m}: m \in \mathbb{N}\right)$ in $X$ such that

$$
\lim _{n \rightarrow \infty} \sup _{m>n} J\left(x_{n}, x_{m}\right)=0
$$

and

$$
\lim _{m \rightarrow \infty} J\left(x_{m}, y_{m}\right)=0,
$$

we have

$$
\lim _{m \rightarrow \infty} d\left(x_{m}, y_{m}\right)=0 .
$$

Remark 2.7 If $(X, d)$ is a $b$-metric space (with $s \geq 1$ ), then the $b$-metric $d: X \times X \rightarrow[0, \infty)$ is a $b$-generalized pseudodistance on $X$. However, there exists a $b$-generalized pseudodistance on $X$ which is not a $b$-metric (for details see Example 4.1 of [16]). 
Remark 2.8 From $(J 1)$ and $(J 2)$ it follows that for any $x, y \in X$ we have $J(x, y)>0$ or $J(y, x)>0$.

By using the notion of $b$-generalized pseudodistance on a $b$-metric space $X$, we can define the $\mathcal{H}^{J}$ Hausdorff distance as below.

Definition 2.9 Let $X$ be a $b$-metric space (with $s \geq 1$ ) and let the map $J: X \times X \rightarrow[0, \infty$ ) be a $b$-generalized pseudodistance on $X$. Let $J(u, V)=\inf _{v \in V} J(u, v)$, where $u \in X$ and $V \in \mathcal{C B}(X)$. Define $\mathcal{H}^{J}: \mathcal{C B}(X) \times \mathcal{C B}(X) \rightarrow[0, \infty)$ by

$$
\mathcal{H}^{J}(A, B)=\max \left\{\sup _{u \in A} J(u, B), \sup _{v \in B} J(v, A)\right\}, \quad \forall A, B \in \mathcal{C B}(X) .
$$

Similarly, the following definitions and notations can be constructed in $b$-metric spaces equipped with a $b$-generalized pseudodistance.

Let $(X, d)$ be a $b$-metric space (with $s \geq 1)$ and let $(A, B)$ be a nonempty pair of subsets of $X$ and let the map $J: X \times X \rightarrow[0, \infty)$ be a $b$-generalized pseudodistance on $X$. We set

$$
\begin{array}{ll}
A_{0}:=\{x \in A: J(x, y)=\operatorname{dist}(A, B), & \text { for some } y \in B\} ; \\
B_{0}:=\{y \in B: J(x, y)=\operatorname{dist}(A, B), & \text { for some } x \in A\} ; \\
J^{*}(a, B):=\frac{1}{S} J(a, B)-\operatorname{dist}(A, B), & \forall a \in A .
\end{array}
$$

Definition 2.10 Let $X$ be a $b$-metric space (with $s \geq 1$ ) and let the map $J: X \times X \rightarrow[0, \infty$ ) be a $b$-generalized pseudodistance on $X$. Let $(A, B)$ be a pair of nonempty subsets of $X$ with $A_{0} \neq \emptyset$.

(I) The pair $(A, B)$ is said to have the $W P^{J}$-property if and only if

$$
\left\{\begin{array}{l}
J\left(x_{1}, y_{1}\right)=\operatorname{dist}(A, B), \\
J\left(x_{2}, y_{2}\right)=\operatorname{dist}(A, B)
\end{array} \quad \Rightarrow J\left(x_{1}, x_{2}\right) \leq J\left(y_{1}, y_{2}\right),\right.
$$

where $x_{1}, x_{2} \in A_{0}$ and $y_{1}, y_{2} \in B_{0}$.

(II) We say that the $b$-generalized pseudodistance $J$ is associated with the pair $(A, B)$ if for any sequences $\left(x_{m}: m \in \mathbb{N}\right)$ and $\left(y_{m}: m \in \mathbb{N}\right)$ in $X$ such that $\lim _{m \rightarrow \infty} x_{m}=x$; $\lim _{m \rightarrow \infty} y_{m}=y$; and

$$
J\left(x_{m}, y_{m-1}\right)=\operatorname{dist}(A, B), \quad \forall m \in \mathbb{N},
$$

we have $d(x, y)=\operatorname{dist}(A, B)$.

We mention that for a $b$-metric space $(X, d)$ if we put $J=d$, then the map $d$ is associated with each pair $(A, B)$, where $(A, B)$ is a nonempty pair in $X$ because of the continuity of $d$.

Definition 2.11 Let $(X, \tau)$ be a topological vector space and $(A, B)$ be a nonempty pair of subsets of $X$. The multivalued non-self mapping $T: A \rightarrow 2^{B}$ is called closed whenever $\left(x_{m}: m \in \mathbb{N}\right)$ is a sequence in $A$ converging to $x \in A$ and $\left(y_{m}: m \in \mathbb{N}\right)$ is a sequence in $B$ satisfying the condition

$$
y_{m} \in T\left(x_{m}\right), \quad \forall m \in \mathbb{N},
$$

and converging to $y \in B$, then $y \in T(x)$.

The following lemma will be used in the sequel. 
Lemma 2.12 [16] Let $X$ be a complete b-metric space (with $s \geq 1$ ) equipped with the $b$-generalized pseudodistance $J$ and let the sequence $\left(x_{m}: m \in\{0\} \cup \mathbb{N}\right)$ satisfy

$$
\lim _{n \rightarrow \infty} \sup _{m>n} J\left(x_{n}, x_{m}\right)=0 .
$$

Then $\left(x_{m}: m \in\{0\} \cup \mathbb{N}\right)$ is a Cauchy sequence on $X$.

\section{Main results}

We begin our main result of this section with the following notion.

Definition 3.1 Define a function $\eta$ by (2.1). Let $X$ be a $b$-metric space (with $s \geq 1$ ) and let the map $J: X \times X \rightarrow[0, \infty)$ be a $b$-generalized pseudodistance on $X$. Let $(A, B)$ be a pair of nonempty pair of subsets of $X$. A multivalued non-self mapping $T: A \rightarrow 2^{B}$ is said to be a contraction of Suzuki type with respect to $b$-generalized pseudodistances provided that there exists $r \in[0,1)$ such that

$$
\frac{\eta(r)}{s} J^{*}(x, T x) \leq J(x, y) \text { implies } s \mathcal{H}^{J}(T x, T y) \leq r J(x, y),
$$

for all $x, y \in A$.

It is clear that the class of multivalued non-self mappings which are contraction of Suzuki type with respect to $b$-generalized pseudodistances contains the class of multivalued non-self mappings considered in Theorem 2.3. This can be seen by taking $s=1$ and $J=d$.

We now prove the main result of this article.

Theorem 3.2 Let $X$ be a complete $b$-metric space (with $s \geq 1$ ) and let the map $J: X \times X \rightarrow$ $[0, \infty)$ be a b-generalized pseudodistance on $X$. Let $(A, B)$ be a pair of nonempty closed subsets of $X$ with $A_{0} \neq \emptyset$ and such that $(A, B)$ has the $W P^{J}$-property and $J$ is associated with $(A, B)$. Let $T: A \rightarrow 2^{B}$ be a closed contraction multivalued non-self mapping of Suzuki type. If $T(x) \in \mathcal{C B}(X)$ for all $x \in A$, and $T(x) \subset B_{0}$ for each $x \in A_{0}$, then $T$ has a best proximity point in $A$.

Proof Choose a real number $r_{1}$ with $0 \leq r<r_{1}<1$. Let $x_{0} \in A_{0}, y_{0} \in T x_{0} \subseteq B_{0}$. Then there exists $x_{1} \in A_{0}$ such that

$$
J\left(x_{1}, y_{0}\right)=\operatorname{dist}(A, B) .
$$

Since

$$
J\left(x_{0}, T x_{0}\right) \leq J\left(x_{0}, y_{0}\right) \leq s\left[J\left(x_{0}, x_{1}\right)+J\left(x_{1}, y_{0}\right)\right],
$$

using (3.2), we deduce

$$
\begin{aligned}
J^{*}\left(x_{0}, T x_{0}\right) & =\frac{1}{s} J\left(x_{0}, T x_{0}\right)-\operatorname{dist}(A, B) \leq \frac{1}{s} s\left[J\left(x_{0}, x_{1}\right)+J\left(x_{1}, y_{0}\right)\right]-\operatorname{dist}(A, B)= \\
& =J\left(x_{0}, x_{1}\right)+J\left(x_{1}, y_{0}\right)-\operatorname{dist}(A, B)=J\left(x_{0}, x_{1}\right),
\end{aligned}
$$

which implies that $\frac{1}{s} J^{*}\left(x_{0}, T x_{0}\right) \leq J^{*}\left(x_{0}, T x_{0}\right) \leq J\left(x_{0}, x_{1}\right)$. Since $\eta(r) \leq 1$, we obtain

$$
\frac{\eta(r)}{s} J^{*}\left(x_{0}, T x_{0}\right) \leq J\left(x_{0}, x_{1}\right) .
$$


By the fact that $T$ is multivalued non-self mapping contraction of Suzuki type, we have

$$
s \mathcal{H}^{J}\left(T x_{0}, T x_{1}\right) \leq r J\left(x_{0}, x_{1}\right) .
$$

Thus

$$
J\left(y_{0}, T x_{1}\right) \leq \mathcal{H}^{J}\left(T x_{0}, T x_{1}\right) \leq \frac{r}{s} J\left(x_{0}, x_{1}\right)<\frac{r_{1}}{s} J\left(x_{0}, x_{1}\right) .
$$

Therefore, there exists $y_{1} \in T x_{1}$ such that

$$
J\left(y_{0}, y_{1}\right) \leq \frac{r_{1}}{s} J\left(x_{0}, x_{1}\right) .
$$

Again, since $T x_{1} \subseteq B_{0}$, there exists an element $x_{2} \in A_{0}$ such that

$$
J\left(x_{2}, y_{1}\right)=\operatorname{dist}(A, B) \text {. }
$$

Also,

$$
J\left(x_{1}, T x_{1}\right) \leq J\left(x_{1}, y_{1}\right) \leq s\left[J\left(x_{1}, x_{2}\right)+J\left(x_{2}, y_{1}\right)\right],
$$

which implies that

$$
\frac{\eta(r)}{s} J^{*}\left(x_{1}, T x_{1}\right) \leq J\left(x_{1}, x_{2}\right)
$$

So,

$$
s \mathcal{H}^{J}\left(T x_{1}, T x_{2}\right) \leq r J\left(x_{1}, x_{2}\right)
$$

Therefore,

$$
J\left(y_{1}, T x_{2}\right) \leq \mathcal{H}^{J}\left(T x_{1}, T x_{2}\right) \leq \frac{r}{s} J\left(x_{1}, x_{2}\right)<\frac{r_{1}}{s} J\left(x_{1}, x_{2}\right) .
$$

Hence,

$$
J\left(y_{1}, y_{2}\right) \leq \frac{r_{1}}{s} J\left(x_{1}, x_{2}\right) .
$$

Continuing this process, by induction, we can find sequences $\left(x_{m}: m \in\{0\} \cup \mathbb{N}\right)$ and $\left(y_{m}: m \in\{0\} \cup \mathbb{N}\right)$ such that

- $x_{m} \in A_{0}$ and $y_{m} \in B_{0}$ for all $m \in\{0\} \cup \mathbb{N}$,

- $y_{m} \in T x_{m}$ for all $m \in\{0\} \cup \mathbb{N}$,

- $J\left(x_{m}, y_{m-1}=\operatorname{dist}(A, B)\right.$ for all $m \in \mathbb{N}$,

- $J\left(y_{m-1}, y_{m}\right) \leq \frac{r_{1}}{s} J\left(x_{m-1}, x_{m}\right)$ for all $m \in \mathbb{N}$.

Now, for any $m \in \mathbb{N}$ we have $J\left(x_{m}, y_{m-1}\right)=\operatorname{dist}(A, B)$ and $J\left(x_{m+1}, y_{m}\right)=\operatorname{dist}(A, B)$. Since $(A, B)$ satisfies the $\mathrm{WP}^{J}$-property, we conclude that

$$
J\left(x_{m}, x_{m+1}\right) \leq J\left(y_{m-1}, y_{m}\right), \quad \forall m \in \mathbb{N} .
$$

Thereby,

$$
\begin{aligned}
J\left(x_{m}, x_{m+1}\right) & \leq J\left(y_{m-1}, y_{m}\right) \leq \frac{r_{1}}{s} J\left(x_{m-1}, x_{m}\right) \leq \frac{r_{1}}{s} J\left(y_{m-2}, y_{m-1}\right) \\
& \leq\left(\frac{r_{1}}{s}\right)^{2} J\left(x_{m-2}, x_{m-1}\right) \leq\left(\frac{r_{1}}{s}\right)^{2} J\left(y_{m-3}, y_{m-2}\right) \leq\left(\frac{r_{1}}{s}\right)^{3} J\left(x_{m-3}, x_{m-2}\right) \\
& \leq \cdots \leq\left(\frac{r_{1}}{s}\right)^{m} J\left(y_{0}, y_{1}\right) \leq\left(\frac{r_{1}}{s}\right)^{m+1} J\left(x_{0}, x_{1}\right) .
\end{aligned}
$$


Now, for each $m>n$ we have

$$
\begin{aligned}
J\left(x_{n}, x_{m}\right) & \leq s\left[J\left(x_{n}, x_{n+1}\right)+J\left(x_{n+1}, x_{m}\right)\right] \\
& \leq s J\left(x_{n}, x_{n+1}\right)+s^{2}\left[J\left(x_{n+1}, x_{n+2}\right)+J\left(x_{n+2}, x_{m}\right)\right] \\
& =s J\left(x_{n}, x_{n+1}\right)+s^{2} J\left(x_{n+1}, x_{n+2}\right)+s^{2} J\left(x_{n+2}, x_{m}\right) \\
& \leq \cdots \leq \sum_{k=0}^{m-(n+1)} s^{k+1} J\left(x_{n+k}, x_{n+k+1}\right) \leq \sum_{k=0}^{m-(n+1)} s^{k+1}\left(\frac{r_{1}}{s}\right)^{k+n+1} J\left(x_{0}, x_{1}\right) \\
& \leq\left(\frac{r_{1}}{s}\right)^{n} \sum_{k=0}^{m-(n+1)} r_{1}^{k+1} J\left(x_{0}, x_{1}\right)
\end{aligned}
$$

Thus, as $n \rightarrow \infty$ in above relation, we deduce that

$$
\lim _{n \rightarrow \infty} \sup _{m>n} J\left(x_{n}, x_{m}\right)=0 .
$$

Similar calculation implies that

$$
\forall_{n>m}\left\{J\left(y_{n}, y_{m}\right) \leq \sum_{k=0}^{m-(n+1)} r_{1} s^{k} J\left(x_{n+k}, x_{n+k+1}\right) \leq\left(\frac{r_{1}}{s}\right)^{n+1} \sum_{k=0}^{m-(n+1)} r_{1}^{k} J\left(x_{0}, x_{1}\right)\right\} .
$$

Hence, $\lim _{n \rightarrow \infty} \sup _{m>n} J\left(y_{n}, y_{m}\right)=0$. It now follows from Lemma 2.12 that $\left(x_{m}: m \in\right.$ $\{0\} \cup \mathbb{N})$ and $\left(y_{m}: m \in\{0\} \cup \mathbb{N}\right)$ are Cauchy sequence in $A$ and $B$ respectively. Since $(A, B)$ is a closed pair of subsets of the complete metric b-space $X$, there exists $p \in A$ and $q \in B$ such that $x_{m} \rightarrow p$ and $y_{m} \rightarrow q$. Besides, since $y_{m} \in T x_{m}$ for all $m \in\{0\} \cup \mathbb{N}$ by closedness of $T$, we obtain $q \in T p$. On the other hand, since $J\left(x_{m}, y_{m-1}\right)=\operatorname{dist}(A, B)$ and $J$ is associated with $(A, B)$, we conclude that $d(p, q)=\operatorname{dist}(A, B)$. We now have

$$
\operatorname{dist}(A, B) \leq \mathcal{D}(p, B) \leq \mathcal{D}(p, T p) \leq d(p, q)=\operatorname{dist}(A, B),
$$

that is, $\mathcal{D}(p, T p)=\operatorname{dist}(A, B)$ and so, $p \in A$ is a best proximity point of the non-self mapping $T$.

Next corollaries are obtained from Theorem 3.2.

Corollary 3.3 Let $X$ be a complete b-metric space (with $s \geq 1)$. Let $(A, B)$ be a pair of nonempty closed subsets of $X$ with $A_{0} \neq \varnothing$ and such that $(A, B)$ has the WP-property. Let $T: A \rightarrow 2^{B}$ be a closed contraction multivalued non-self mapping of Suzuki type. If $T(x) \in \mathcal{C B}(X)$ for all $x \in A$, and $T(x) \subset B_{0}$ for each $x \in A_{0}$, then $T$ has a best proximity point in $A$.

Corollary 3.4 (Compare with Theorem 2.3) Let $(A, B)$ be a pair of nonempty closed subsets of a complete metric space $(X, d)$ such that $A_{0} \neq \varnothing$ and $(A, B)$ satisfies the WP-property. Let $T: A \rightarrow 2^{B}$ be a closed contraction multivalued non-self mapping of Suzuki type. If $T(x) \in \mathcal{C B}(X)$ for all $x \in A$, and $T\left(x_{0}\right) \subset B_{0}$ for each $x_{0} \in A_{0}$, then $T$ has a best proximity point in $A$.

Corollary 3.5 Let $X$ be a complete $b$-metric space (with $s \geq 1$ ) and let the map $J: X \times X \rightarrow$ $[0, \infty)$ be a b-generalized pseudodistance on $X$. Let $(A, B)$ be a pair of nonempty closed subsets of $X$ with $A_{0} \neq \varnothing$ and such that $(A, B)$ has the $W P^{J}$-property and $J$ is associated with $(A, B)$. Let $T: A \rightarrow B$ be a continuous and contraction single-valued non-self mapping of Suzuki type. If $T\left(A_{0}\right) \subseteq B_{0}$, then $T$ has a best proximity point in $A$. 
Corollary 3.6 Let $X$ be a complete $b$-metric space (with $s \geq 1$ ). Let $(A, B)$ be a pair of nonempty closed subsets of $X$ with $A_{0} \neq \varnothing$ and such that $(A, B)$ has the WP-property. Let $T: A \rightarrow B$ be a continuous and contraction single-valued non-self mapping of Suzuki type. If $T\left(A_{0}\right) \subseteq B_{0}$, then $T$ has a best proximity point in $A$.

\section{Examples illustrating Theorem 3.2 and some comparisons}

In this section, we will present some examples illustrating the concepts having been introduced so far. We will show a fundamental difference between Theorems 3.2 and 2.3. The examples will show that Theorem 3.2 is an essential generalization of Theorem 2.3. First, we present an example of generalized pseudodistance in metric spaces and $b$-metric spaces, respectively.

Example 4.1 Let $X$ be a metric space ( $b$-metric space respectively) where the metric $d$ : $X \times X \rightarrow[0, \infty)$ is of the form $d(x, y)=|x-y|\left(d(x, y)=|x-y|^{2}\right), x, y \in X$. Let the closed set $E \subset X$, containing at least two different points, be arbitrary and fixed. Let $c>0$ such that $c>\delta(E)$, where $\delta(E)=\sup \{d(x, y): x, y \in X\}$ be arbitrary and fixed. Define the map $J: X \times X \rightarrow[0, \infty)$ as follows:

$$
J(x, y)= \begin{cases}d(x, y) & \text { if }\{x, y\} \cap E=\{x, y\} \\ c & \text { if }\{x, y\} \cap E \neq\{x, y\} .\end{cases}
$$

Then $J: X \times X \rightarrow[0, \infty)$ is generalized pseudodistance on $X$ [17] ( $b$-generalized pseudodistance on $X[16]$.

Next, we present an example which illustrate Theorem 3.2. To compare our results with some well-known best proximity point theorems in the literature, we start by giving an example where $X$ is a metric space.

Example 4.2 Let $(X, d)$ be a metric space, where $X=\mathbb{R}, d(x, y)=|x-y|, x, y \in X$. Let $(A, B)$ be a pair of subsets of $X$, where $A=[0,1] \cup[3,4]$ and $B=[3 / 2,5 / 2]$. Let $E=[0,1] \cup B=[0,1] \cup[3 / 2,5 / 2]$ and let $J: X \times X \rightarrow[0, \infty)$ be defined by the formula

$$
J(x, y)=\left\{\begin{array}{ll}
d(x, y) & \text { if } E \cap\{x, y\}=\{x, y\} \\
4 & \text { if } E \cap\{x, y\} \neq\{x, y\}
\end{array}, \quad x, y \in X .\right.
$$

From Example 4.1, the map $J$ is generalized pseudodistances. Assume that $T: A \rightarrow B$ is of the form

$$
T x= \begin{cases}3 / 2 & \text { if } x \in[0,1] \\ x-1 & \text { if } x \in[3,7 / 2) \\ 5 / 2 & \text { if } x \in[7 / 2,4]\end{cases}
$$

I. We show that pair $(A, B)$ has the $W P^{J}$-property.

Indeed, we observe that $\operatorname{dist}(A, B)=1 / 2$ and

$$
\begin{aligned}
& A_{0}=\{x \in A: J(x, y)=\operatorname{dist}(A, B) \text { for some } y \in B\}=\{1\}, \\
& B_{0}=\{y \in B: J(x, y)=\operatorname{dist}(A, B) \text { for some } x \in A\}=\{3 / 2\} .
\end{aligned}
$$

Hence, the pair $(A, B)$ has the $W P^{J}$-property.

II. We see that $A$ is complete and by (4.2) we have $T\left(A_{0}\right)=T(\{1\})=3 / 2 \in B_{0}$. 
III. We see that $T$ is contraction of Banach type (i.e. $J(T x, T y) \leq r J(x, y)$ for some $r \in[0,1)$ and for all $x, y \in A)$.

Indeed, let $r=1 / 2$ and let $x, y \in A$ be arbitrary and fixed. We see that by (4.2)

$$
T x \in E, \quad \forall x \in A .
$$

We consider the following cases:

Case 1 If $x, y \in[0,1]$, then by (4.2), $T x=T y=3 / 2$. Now, by (4.1) we have:

$$
J(T x, T y)=J(3 / 2,3 / 2)=0 \leq r d(x, y)=r J(x, y) .
$$

Case 2 If $\{x, y\} \cap[3,4] \neq \emptyset$, then by (4.3), $\{T x, T y\} \cap E=\{T x, T y\}$ which, by (4.1) gives $J(T x, T y)=d(T x, T y)$. Moreover, since $\{x, y\} \cap E \neq\{x, y\}$, by (4.1), we obtain $J(x, y)=4$. Hence

$$
J(T x, T y)=d(T x, T y) \leq 1<2=r \cdot 4=r J(x, y) .
$$

In consequence (4.4) and (4.5) implies that $T$ is contraction of Banach type.

IV. We see that $T$ is contraction of Suzuki type (when $s=1$, and $T$ is single valued), i.e.

$$
\frac{1}{1+r} J^{*}(x, T x) \leq J(x, y) \text { implies } J(T x, T y) \leq r J(x, y),
$$

for some $r \in[0,1)$ and for all $x, y \in A$. It is consequence of Step III.

V. We see that there exists a best proximity point of $T$.

Indeed, for $z=1$ we have $d(z, T(z))=d(1,3 / 2)=1 / 2=\operatorname{dist}(A, B)$.

Now, we will compare our results with two important results which existing in the literature. In 2013, Zhang et al. [18] proved the following theorem.

Theorem 4.1 [18] Let $(A, B)$ be a pair of subsets of a metric space $(X, d)$. Let $T$ be a contraction from $A$ into $B$, i.e.

$$
d(T x, T y) \leq r d(x, y),
$$

for some $r \in[0,1)$ and for all $x, y \in A$. Assume that the following hold:

(i) $(A, B)$ has the WP-property.

(ii) $A$ is complete.

(iii) $T\left(A_{0}\right) \subset B_{0}$.

Then there exists a unique $z \in A$ such that $d(z, T z)=d(A, B)$.

In this same year, Suzuki [19] established the following interesting result.

Theorem 4.2 [19] Let $(A, B)$ be a pair of subsets of a metric space $(X, d)$. Let $T$ be a mapping from A into B. Assume that (i)-(iii) in Theorem 4.1 and the following hold:

(iv) There exists $\alpha \in[0,1 / 2)$ such that

$$
d(T x, T y) \leq \alpha(d(x, T x)-\operatorname{dist}(A, B))+\alpha(d(y, T y)-\operatorname{dist}(A, B))),
$$

for all $x, y \in A$. Then there exists a unique $z \in A$ so that $d(z, T z)=d(A, B)$.

Remark 4.3 Let $X, A, B, T$ be as in Example 4.2.

I. We see that the map $T$ is not contraction in the sense of Theorem 4.1. 
Proof Suppose that for $T$ the following condition holds

$$
d(T x, T y) \leq r d(x, y),
$$

for some $r \in[0,1)$ and for all $x, y \in A$. In particular, for $x_{0}=\frac{55}{16}$ and $y_{0}=\frac{7}{2}$ we have $d\left(x_{0}, y_{0}\right)=\frac{1}{16}$ and $d\left(T x_{0}, T y_{0}\right)=d\left(\frac{39}{16}, \frac{5}{2}\right)=\frac{1}{16}$. Hence, and by (4.6) we get

$$
\frac{1}{16}=d\left(T x_{0}, T y_{0}\right) \leq r d\left(x_{0}, y_{0}\right)=r \frac{1}{16}<\frac{1}{16},
$$

which is impossible.

II. We see that the mapping $T$ is not contraction in the sense of Theorem 4.2. In this order, suppose the following condition holds

$$
d(T x, T y) \leq \alpha[d(x, T x)-\operatorname{dist}(A, B)]+\alpha[d(y, T y)-\operatorname{dist}(A, B)],
$$

for some $\alpha \in\left[0, \frac{1}{2}\right)$ and for all $x, y \in A$. In particular, for $x_{0}=\frac{1}{2}$ and $y_{0}=\frac{7}{2}$ we have $d\left(x_{0}, T x_{0}\right)=d\left(\frac{1}{2}, \frac{3}{2}\right)=1, d\left(y_{0}, T y_{0}\right)=d\left(\frac{7}{2}, \frac{5}{2}\right)=1$ and $d\left(T x_{0}, T y_{0}\right)=d\left(\frac{3}{2}, \frac{5}{2}\right)=$ 1. Hence, and by (4.7) we get

$$
\begin{aligned}
1 & =d\left(T x_{0}, T y_{0}\right) \leq \alpha\left[d\left(x_{0}, T x_{0}\right)-\operatorname{dist}(A, B)\right]+\alpha\left[d\left(y_{0}, T y_{0}\right)-\operatorname{dist}(A, B)\right] \\
& =\alpha\left[1-\frac{1}{2}\right]+\alpha\left[1-\frac{1}{2}\right]=\alpha<\frac{1}{2},
\end{aligned}
$$

which is impossible.

Remark 4.4 It is worth noticing that the concept of generalized pseudodistances gives that the $P$-property and $P^{J}$-property are different. In Example 4.2, we proved that the pair $(A, B)$ has the $P^{J}$-property. However, we observe that the pair $(A, B)$ does not have the $P$-property or even the $W P$-property. Indeed, for usual metric $d$ we have $A_{0}=\{1,3\}$ and $B_{0}=\{3 / 2,5 / 2\}$. Hence

$$
\begin{aligned}
& d(1,3 / 2)=1 / 2 \\
& d(3,5 / 2)=1 / 2
\end{aligned},
$$

however $2=d(1,3)>d(3 / 2,5 / 2)=1$. Thus the pair $(A, B)$ does not have the $P$-property and $W P$-property.

Remark 4.5 In 2013, Abkar and Gabeleh [20] proved that some recent results concerning the existence of best proximity points can be obtained from the same results in fixed point theory. The Authors used a bijective isometry $g: A_{0} \rightarrow B_{0}$ such that $d(x, g(x))=\operatorname{dist}(A, B)$ (see Theorem 10 of [20]). It is worth noticing, that in our results such kind consideration is not true. Indeed, the fact $J(x, y)=\operatorname{dist}(A, B), J\left(x, y^{\prime}\right)=\operatorname{dist}(A, B)$ and $P^{J}$-property or $W P^{J}$-property does not imply that $J\left(y, y^{\prime}\right)=J\left(y^{\prime}, y\right)=0$ and $y=y^{\prime}$. That would be possible if $\max \{J(x, x), J(x, x)\}=0$. However, in general it does not hold. Moreover, in the literature there are no fixed point theorem for such kind contraction with respect to $J$-generalized pseudodistances. We obtain such kind result as immediate corollary from Theorem 3.2 (see Corollary 3.5).

Example 4.3 Let $(X, d)$ be a $b$-metric space, where $X=\mathbb{R}, d(x, y)=|x-y|^{2}, x, y \in X$. Let $(A, B)$ be a pair of subset $X$, where $A=[0,1] \cup\{4,5\}$ and $B=[6,8]$. Let $E=\{0,1\} \cup[4,8]$ and let $J: X \times X \rightarrow[0, \infty)$ be defined by the formula

$$
J(x, y)=\left\{\begin{array}{ll}
d(x, y) & \text { if } E \cap\{x, y\}=\{x, y\} \\
65 & \text { if } E \cap\{x, y\} \neq\{x, y\}
\end{array}, \quad x, y \in X .\right.
$$


From Example 4.1, the map $J$ is $b$-generalized pseudodistances. Assume that $T: A \rightarrow 2^{B}$ is of the form

$$
T x= \begin{cases}{\left[6, \frac{13}{2}\right] \cup\{7\}} & \text { if } x=0 \\ \{7\} & \text { if } x \in(0,1) \\ \{7\} \cup\left[\frac{15}{2}, 8\right] & \text { if } x=1 \\ \{6\} & \text { if } x \in\{4,5\} .\end{cases}
$$

I. We show that $(A, B)$ has the $W P^{J}$-property.

Indeed, we observe that $\operatorname{dist}(A, B)=1$ and

$$
\begin{aligned}
& A_{0}=\{x \in A: J(x, y)=\operatorname{dist}(A, B) \text { for some } y \in B\}=\{5\}, \\
& B_{0}=\{y \in B: J(x, y)=\operatorname{dist}(A, B) \text { for some } x \in A\}=\{6\} .
\end{aligned}
$$

Hence, the pair $(A, B)$ has the $W P^{J}$-property.

II. We see that $A$ (4.9) we have $T\left(A_{0}\right)=\{6\} \subseteq B_{0}$.

III. We see that $T$ is contraction of Suzuki type with $s=2$, i.e. there exists $r \in[0,1)$ so that

$$
\frac{1}{1+r} J^{*}(x, T x) \leq J(x, y) \text { implies } \mathcal{H}^{J}(T x, T y) \leq r J(x, y),
$$

for all $x, y \in A$. Indeed, let $r=\frac{1}{4}$ and let $x, y \in A$ be arbitrary and fixed. Then by (4.9), we may consider the following cases:

Case 1. If $T x=\left[6, \frac{13}{2}\right] \cup\{7\}, T y=\{7\} \cup\left[\frac{15}{2}, 8\right]$, then $x=0, y=1$ and $\mathcal{H}^{J}(T x, T y)=1$. Moreover, by (4.8), we calculate: $J(x, T x)=J\left(0,\left[6, \frac{13}{2}\right] \cup\right.$ $\{7\})=36 ; J^{*}(x, T x)=\frac{1}{2} J(x, T x)-\operatorname{dist}(A, B)=17$. Hence, $\frac{1}{1+r} J^{*}(x, T x)=$ $\frac{68}{5} \geq 1=J(x, y)$, which gives that in this case the condition (4.10) holds.

Case 2. If $T x=\{7\} \cup\left[\frac{15}{2}, 8\right], T y=\left[6, \frac{13}{2}\right] \cup\{7\}$, then $x=1, y=$ 0 and $\mathcal{H}^{J}(T x, T y)=1$. Moreover, by (4.8), we calculate: $J(x, T x)=$ $J\left(1,\{7\} \cup\left[\frac{15}{2}, 8\right]\right)=36 ; J^{*}(x, T x)=\frac{1}{2} J(x, T x)-\operatorname{dist}(A, B)=17$. Hence, $\frac{1}{1+r} J^{*}(x, T x)=\frac{68}{5} \geq 1=J(x, y)$, which gives that in this case the condition (4.10) holds.

Case 3. If $T x=\left[6, \frac{13}{2}\right] \cup\{7\}$ and $T y=\{7\}$, then $x=0, y \in(0,1) \cup\{4\}$ and $\mathcal{H}^{J}(T x, T y)=1$. Moreover, by (4.8), we calculate: $J(x, y)=65$ if $y \in(0,1)$ (since $(0,1) \cap E=\emptyset)$ or $J(x, y)=d(x, y)=16$ if $y=4 ; s \mathcal{H}^{J}(T x, T y)=2 \leq \frac{1}{4} J(x, y)$, which gives that in this case the condition (4.10) holds.

Case 4. If $T x=\left[6, \frac{13}{2}\right] \cup\{7\}$ and $T y=\{6\}$, then $x=0, y \in\{4,5\}$ and $\mathcal{H}^{J}(T x, T y)=1$. Moreover, by (4.8), we calculate: $J(x, y)=16$ (if $y=4$ ) or $J(x, y)=25$ (if $y=5$ ); in both cases we get $s \mathcal{H}^{J}(T x, T y)=2 \leq \frac{1}{4} J(x, y)$, which gives that in this case the condition (4.10) holds.

Case 5. If $T x=\{7\} \cup\left[\frac{15}{2}, 8\right]$ and $T y=\{6\}$, then $x=1, y \in\{4,5\}$ and $\mathcal{H}^{J}(T x, T y)=1$. Moreover, by (4.8), we calculate: $J(x, y)=16$ (if $y=4$ ) or $J(x, y)=25$ (if $y=5$ ); in both cases we get $s \mathcal{H}^{J}(T x, T y)=2 \leq \frac{1}{4} J(x, y)$, which gives that in this case the condition (4.10) holds.

Case 6. If $T x=\{7\} \cup\left[\frac{15}{2}, 8\right]$ and $T y=\{7\}$, then $x=1, y \in(0,1)$ and $\mathcal{H}^{J}(T x, T y)=1$. Moreover, by (4.8), we calculate: $J(x, y)=65$ (since $(0,1) \cap E=\emptyset) ; s \mathcal{H}^{J}(T x, T y)=2 \leq \frac{1}{4} J(x, y)$, which gives that in this case the condition (4.10) holds. 
Case 7. If $T x=\{7\}, T y=\{6\}$, then $x \in(0,1), y \in\{4,5\}$ and $\mathcal{H}^{J}(T x, T y)=1$. Moreover, by (4.8), we calculate: $J(x, y)=65$ (since $(0,1) \cap E=\emptyset$ ) and then $s \mathcal{H}^{J}(T x, T y)=2 \leq \frac{1}{4} J(x, y)$, which gives that in this case the condition (4.10) holds.

Case 8. If $T x=\{6\}, T y=\{7\}$, then $x_{1}\{4,5\}, y \in(0,1)$ and $\mathcal{H}^{J}(T x, T y)=1$. Moreover, by (4.8), we calculate: $J(x, y)=65$ if $y \in(0,1)$ (since $(0,1) \cap E=\emptyset$ ) and then $s \mathcal{H}^{J}(T x, T y)=2 \leq \frac{1}{4} J(x, y)$, which gives that in this case the condition (4.10) holds.

In consequence, using the symmetry of $J$, we conclude that the map $T$ is contraction of Suzuki type.

IV. We see that there exists a best proximity point of $T$.

Indeed, for $z=5$ we have $d(z, T z)=d(5,\{6\})=1=\operatorname{dist}(A, B)$.

Now, we will compare our result with another result for $J$-generalized pseudodistance [16].

Theorem 4.6 [16] Let $X$ be a complete b-metric space (with $s \geq 1$ ) and let the map $J$ : $X \times X \rightarrow[0, \infty)$ be a b-generalized pseudodistance on $X$. Let $(A, B)$ be a pair of nonempty closed subsets of $X$ with $A_{0} \neq \emptyset$ and such that $(A, B)$ has the $P^{J}$-property and $J$ is associated with $(A, B)$. Let $T: A \rightarrow 2^{B}$ be a closed and contraction multivalued non-self mapping of Nadler type i.e.

$$
s \mathcal{H}^{J}(T x, T y) \leq \lambda J(x, y),
$$

for some $\lambda \in[0,1)$ and for all $x, y \in A$. If $T(x)$ is bounded and closed in $B$ for all $x \in A$, and $T(x) \subset B_{0}$ for each $x \in A_{0}$, then $T$ has a best proximity point in $A$.

Remark 4.7 Let $X, A, B, T, E$ and $J$ be as in Example 4.3. We see that the map $T$ is not contraction of Nadler type (in sense of Theorem 4.6.)

Proof To this end, suppose there exists $\lambda \in[0,1)$ so that the following condition holds

$$
s \mathcal{H}^{J}(T x, T y) \leq \lambda J(x, y), \quad \forall x, y \in A .
$$

In particular, for $x_{0}=0$ and $y_{0}=1$, by (4.8) we have $J\left(x_{0}, y_{0}\right)=1$ and $\mathcal{H}^{J}\left(T x_{0}, T y_{0}\right)=1$. Hence, by (4.10), we get

$$
2=s \mathcal{H}^{J}\left(T x_{0}, T y_{0}\right) \leq \lambda J\left(x_{0}, y_{0}\right)=\lambda \cdot 1<1,
$$

which is impossible.

Open Access This article is distributed under the terms of the Creative Commons Attribution 4.0 International License (http://creativecommons.org/licenses/by/4.0/), which permits unrestricted use, distribution, and reproduction in any medium, provided you give appropriate credit to the original author(s) and the source, provide a link to the Creative Commons license, and indicate if changes were made.

\section{References}

1. Suzuki, T.: A generalized Banach contraction principle which characterizes metric completeness. Proc. Am. Math. Soc. 136(5), 1861-1869 (2008)

2. Kikkawa, M., Suzuki, T.: Some similarity between contractions and Kannan mappings. Fixed Point Theory Appl., Article ID 649749, 1-8 (2010) 
3. Kirk, W.A., Reich, S., Veeramani, P.: Proximinal retracts and best proximity pair theorems. Numer. Funct. Anal. Optim. 24, 851-862 (2003)

4. Abkar, A., Gabeleh, M.: The existence of best proximity points for multivalued non-self-mappings. RACSAM 107, 319-325 (2013)

5. Amini-Harandi, A.: Best proximity points theorems for cyclic strongly quasi-contraction mappings. J. Glob. Optim. 56, 1667-1674 (2013)

6. Gabeleh, M.: Best proximity points: global minimization of multivalued non-self mappings. Optim. Lett. 8, 1101-1112 (2014)

7. Sadiq Basha, S., Shahzad, N., Jeyaraj, R.: Best proximity point theorems: exposition of a significant non-linear programming problem. J. Glob. Optim. 56, 1699-1705 (2013)

8. Sadiq Basha, S.: Best proximity points: global optimal approximate solutions. J. Glob. Optim. 49, 15-21 (2011)

9. Wlodarczyk, K., Plebaniak, R., Banach, A.: Best proximity points for cyclic and noncyclic set-valued relatively quasi-asymptotic contractions in uniform spaces. Nonlinear Anal. 70, 3332-3342 (2009)

10. Wlodarczyk, K., Plebaniak, R., Banach, A.: Erratum to: Best proximity points for cyclic and noncyclic set-valued relatively quasi-asymptotic contractions in uniform spaces. Nonlinear Anal. 71, 3583-3586 (2009)

11. Sankar Raj, V.: A best proximity point theorem for weakly contractive non-self-mappings. Nonlinear Anal. 74, 4804-4808 (2011)

12. Gabeleh, M.: Global optimal solutions of non-self mappings. U.P.B. Sci. Bull. Ser. A 75, 67-74 (2013)

13. Abkar, A., Gabeleh, M.: Global optimal solutions of noncyclic mappings in metric spaces. J. Optim. Theory Appl. 153, 298-305 (2012)

14. Gabeleh, M.: Best proximity point theorems for single- and set-valued non-self Mappings. Acta Math. Sci. 34B, 1661-1669 (2014)

15. Czerwik, S.: Nonlinear set-valued contraction mappings in $b$-metric spaces. Atti Sem. Mat. Fis. Univ. Modena 46, 263-276 (1998)

16. Plebaniak, R.: On best proximity points for set-valued contractions of Nadler type with respect to bgeneralized pseudodistances in b-metric spaces. Fixed Point Theory Appl. 2014, 39 (2014). doi:10.1186/ 1687-1812-2014-39

17. Wlodarczyk, K., Plebaniak, R.: Contractions of Banach, Tarafdar, Meir-Keeler, Ćirić-JachymskiMatkowski and Suzuki types and fixed points in uniform spaces with generalized pseudodistances. J. Math. Anal. Appl. 404(2), 338-350 (2013)

18. Zhang, J., Su, Y., Cheng, Q.: A note on 'A best proximity point theorem for Geraghty-contractions'. Fixed Point Theory Appl. 2013, Article ID 99 (2013)

19. Suzuki, T.: The existence of best proximity points with the weak P-property. Fixed Point Theory Appl. 2013, 259 (2013)

20. Abkar, A., Gabeleh, M.: A note on some best proximity point theorems proved under P-property. Abstract Appl. Anal. 2013, Article ID 189567, 3 pp 